\title{
Homogeneous Graft Copolymerization of Vinyl Monomers onto Cellulose in a Dimethyl Sulfoxide-Paraformaldehyde Solvent System II. Characterization of Graft Copolymers
}

\author{
Noboru NishioKa, Keiji MATSUmoto, and Kouichi KoSAI \\ Faculty of Engineering, Osaka Electro-Communication University, \\ Neyagawa 572, Japan
}

(Received September 27, 1982)

\begin{abstract}
Homogeneous graft copolymerization of acrylonitrile and of methyl methacrylate onto cellulose using radical initiators was carried out in a dimethyl sulfoxideparaformaldehyde solvent system. The graft copolymers obtained under various grafting conditions were hydrolyzed to isolate the graft polymers. The molecular weight of graft polymer and the number of grafts per cellulose chain were determined as functions of polymerization temperature and concentrations of monomer and initiator. The molecular weight of graft polymer increased with increasing monomer concentration but decreased with raising temperature and with increasing initiator concentration. The number of grafts increased with raising temperature and with increasing initiator concentration but decreased with increasing monomer concentration.

KEY WORDS Homogeneous Grafting / Cellulose / Methyl Methacrylate /

Acrylonitrile / Dimethyl Sulfoxide-Paraformaldehyde Solvent System /

Hydrolysis / Molecular Weight of Graft Polymer / Number of Grafts /
\end{abstract}

Graft copolymerization of vinyl monomers onto cellulose is usually carried out in a heterogeneous solvent system because of the lack of relevant solvent systems for cellulose. ${ }^{1-4}$ It is considered that grafting in such a heterogeneous system proceeds only in amorphous regions, causing the number of grafts per cellulose chain seldom to exceed unity. ${ }^{5-9}$

In the previous paper, ${ }^{10}$ dimethyl sulfoxide (DMSO) containing paraformaldehyde (PF) was used as a solvent for cellulose and the homogeneous grafting of acrylonitrile (AN) and of methyl methacrylate (MMA) onto cellulose using radical initiators was carried out under various grafting conditions to determine the optimum conditions for each monomer-initiator system. Furthermore, the graft copolymers obtained under the optimum conditions were hydrolyzed to isolate the graft polymers and the number of grafts was found to be more than unity. The homogeneous grafting of styrene onto cellulose in a sulfur dioxidediethylamine-DMSO solvent system has also in- dicated that the number of grafts was in the range from 2.4 to $10.6 .{ }^{11}$ Probable reason for these findings is that, in the homogeneous system employed, all the cellulose chains are exposed to chain transfer reactions leading to the formation of cellulose macroradicals.

The present study deals with the characterization of the graft copolymers obtained under various grafting conditions. The molecular weight of the graft polymer and the number of grafts per cellulose chain were determined as functions of polymerization temperature and concentrations of monomer and initiator. The knowledge about the structure of graft copolymers will serve to elucidate the mechanism of graft copolymerization.

\section{EXPERIMENTAL}

\section{Hydrolysis}

The graft products prepared in our previous work $^{10}$ were used for this study. The crude graft products were treated in a Soxhlet apparatus for 
more than $24 \mathrm{~h}$ to extract attendant homopolymers with dimethylformamide (DMF) and acetone for poly(acrylonitrile) (PAN) and poly(methyl methacrylate) (PMMA), respectively. The graft products subjected to extraction were hydrolyzed to isolate the graft polymers.

The graft PAN was isolated by the following procedure. ${ }^{12} \mathrm{~A}$ given sample was oxidized by immersing it in aqueous potassium periodate at room temperature for more than $48 \mathrm{~h}$. After filtration, the residue was immersed in $2.5 \mathrm{~N}$ sodium hydroxide at room temperature for more than $48 \mathrm{~h}$. The remaining residue was obtained by filtration, dried, and dissolved in DMF. Then the solution was filtered to collect the filtrate, which was evaporated to recover graft PAN.

Hydrolysis of the cellulose grafted with MMA was carried out by immersing the sample in $72 \%$ $\mathrm{H}_{2} \mathrm{SO}_{4}$ for more than $6 \mathrm{~h} .{ }^{13}$ The reaction mixture was then poured into distilled water with vigorous stirring. The precipitate recovered was washed with water repeatedly, dried, and dissolved in acetone. After filtration, the filtrate was evaporated to recover graft PMMA.

\section{Molecular Weight Determination}

The intrinsic viscosities, $[\eta]$ (in $\mathrm{cm}^{3} \mathrm{~g}^{-1}$ ), of the isolated graft polymers and the attendant homopolymers extracted were measured in a Ubbelohde dilution viscometer at $25^{\circ} \mathrm{C}$. The solvents were DMF for PAN and acetone for PMMA. The following equations were used to estimate viscosityaverage molecular weights. ${ }^{14,15}$

$$
\begin{aligned}
& {[\eta]_{\text {DMF }}=24.3 \times 10^{-3} \mathbf{M}^{0.75}} \\
& {[\eta]_{\text {acetone }}=5.3 \times 10^{-3} \mathbf{M}^{0.73}}
\end{aligned}
$$

The molecular weight of the backbone cellulose was estimated to be $16 \times 10^{4}$ from [ $\eta$ ] obtained with the DMSO-PF solvent at $30^{\circ} \mathrm{C}^{16}$ and with the cadoxen solvent at $20^{\circ} \mathrm{C} .{ }^{17}$

\section{RESULTS AND DISCUSSION}

It is well known that a graft copolymer and homopolymer are produced simultaneously in graft copolymerization. ${ }^{1-3}$ In order to characterize a graft copolymer, it is thus necessary to separate the attendant homopolymer from the crude graft product. For cellulosic graft products, this separation is considered comparatively easier than that for other graft products because of the large solubility differences between graft copolymers and attendant homopolymers. ${ }^{1-3}$ However, the difficulties in separating the attendant homopolymers from the crude graft products have been pointed out by many workers. ${ }^{3,5,6,11,18-20}$ Recently, thin layer chromatography (TLC) has been found effective for separating the residue obtained after hydrolysis of the graft product subjected to the extraction of homopolymer. ${ }^{6,20,21}$ Quite recently, it has been report$\mathrm{ed}^{11}$ that the true grafting percentage of the graft products obtained in the homogeneous grafting of styrene onto cellulose was about 0.8 times the apparent grafting percentage, where the apparent grafting percentage means the value calculated from the weight decrease after extraction of the homopolymer.

As mentioned in our previous paper ${ }^{10}$ some of the graft products subjected to the extraction of homopolymer were analyzed by TLC in order to detect any remaining attendant homopolymer. In no case were any spots found associated with the homopolymer. Consequently, the apparent grafting percentage was regarded as the true one in this study. But the grafting percentage may be slightly overestimated since the residue obtained after hydrolysis of the graft product was not analyzed by TLC.

It is generally accepted that the molecular weight of graft polymer is similar to that of the attendant homopolymer in homogeneous grafting. ${ }^{1-3} \mathrm{Re}-$ cently, some different results on molecular weight have been reported, ${ }^{11,22,23}$ but both molecular weights were consistent with each other within experimental errors in this study.

\section{Initiation with Ammonium Persulfate}

Two radical initiators were tested for the grafting of vinyl monomers onto cellulose. The results obtained with ammonium persulfate (APS) are described first below.

Figures 1 and 2 show the effects of polymerization temperature on the grafting of $\mathrm{AN}$ and MMA, respectively. The data on the grafting percentage reported in the previous paper ${ }^{10}$ are also depicted in these figures. For either monomer system, the grafting percentage shows a maximum around $40^{\circ} \mathrm{C}$, indicating that an optimum temperature is present for each monomer system. 


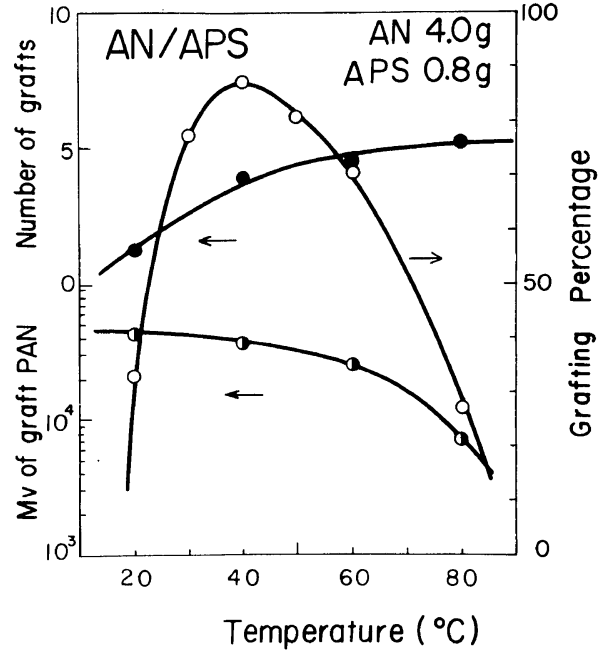

Figure 1. Effect of polymerization temperature on the grafting of AN: (O) grafting percentage; (O) number of grafts; (O) molecular weight of graft polymer; cellulose concn, $2 \%$; grafting time, $5 \mathrm{~h}$; AN, $4 \mathrm{~g} / 100 \mathrm{~g}$ DMSO soln; APS, $0.8 \mathrm{~g} / 100 \mathrm{~g}$ DMSO soln.

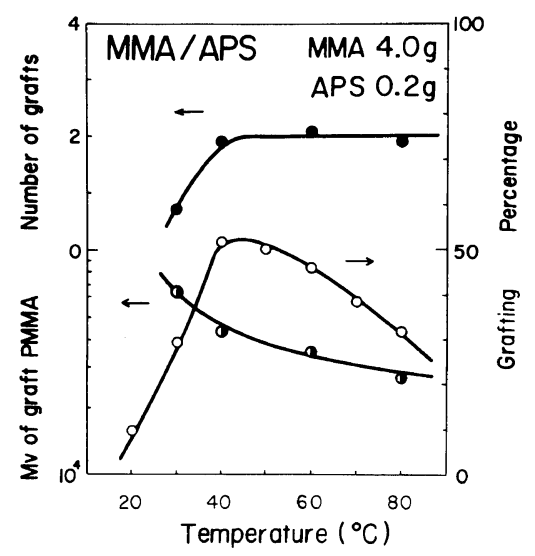

Figure 2. Effect of polymerization temperature on the grafting of MMA: cellulose concn, $2 \%$; grafting time, $3 \mathrm{~h}$.

It is also seen in these figures that for both monomer systems, the molecular weight of graft polymer decreases with raising temperature. This trend is consistent with the expectation based on the kinetics of radical polymerization. ${ }^{3}$ On the other hand, the number of grafts increases with raising temperature and levels off. The decrease in grafting percentage at higher temperatures can be explained by the decrease in the molecular weight of graft

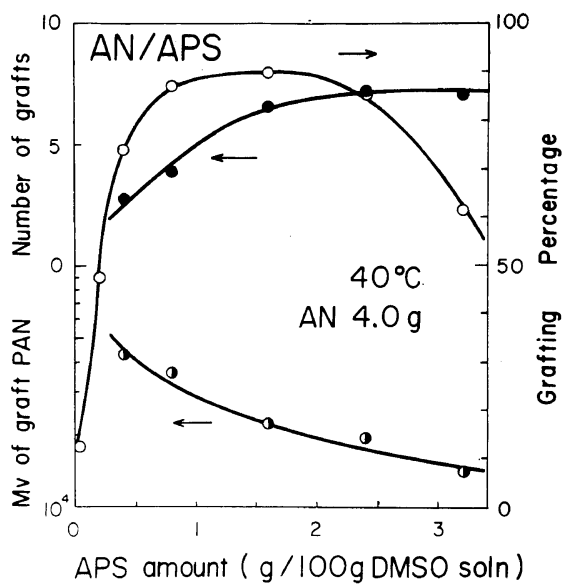

Figure 3. Effect of initiator concentration on the grafting of AN: cellulose concn, $2 \%$; grafting time, $5 \mathrm{~h}$; temp, $40^{\circ} \mathrm{C}$.

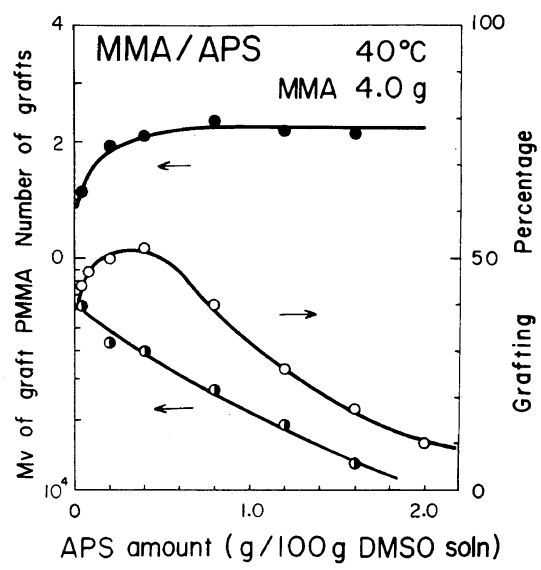

Figure 4. Effect of initiator concentration on the grafting of MMA: cellulose concn, $2 \%$; grafting time, $3 \mathrm{~h}$.

polymer.

Two procedures may be given to produce graft copolymers of high grafting percentage. One is to increase the number of grafts; the other is to increase the molecular weight of graft polymer. Among grafting parameters, the initiator concentration is probably associated with both the number of grafts and the molecular weight of graft polymer. ${ }^{8,24-26}$

Figure 3 illustrates how the grafting of AN was affected by the initiator concentration. The initiator concentration is expressed in grams of ASP in $100 \mathrm{~g}$ of the DMSO solution and all grafting was carried 


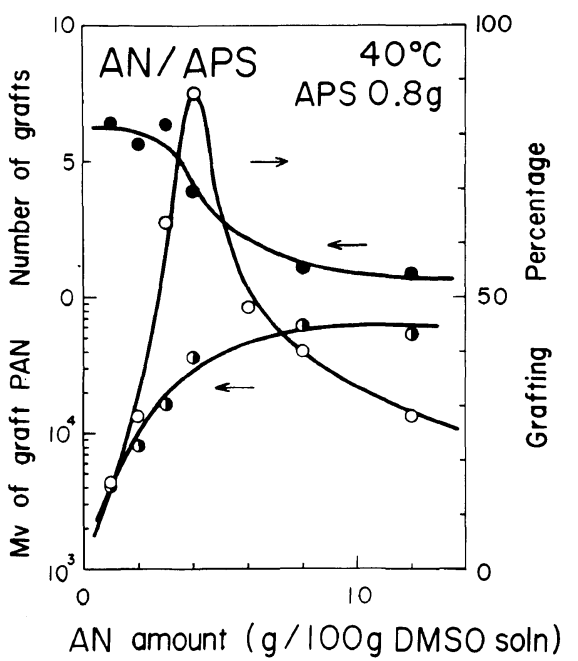

Figure 5. Effect of monomer concentration on the grafting of AN: cellulose concn, $2 \%$; grafting time, $5 \mathrm{~h}$.

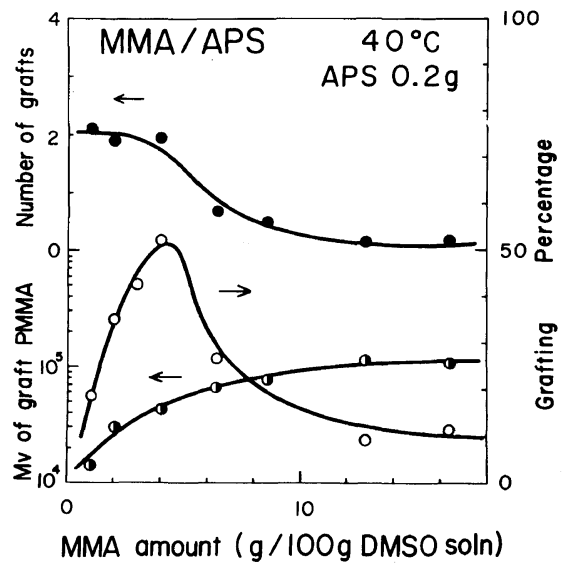

Figure 6. Effect of monomer concentration on the grafting of MMA: cellulose concn, $2 \%$; grafting time $3 \mathrm{~h}$.

out at $40^{\circ} \mathrm{C}$. The molecular weight of graft PAN decreases with increasing APS concentration and the number of grafts increases and reaches up to about 7 at higher concentrations. The leveling off in the number of grafts at higher APS concentrations is ascribed to the equilibration in radical concentration, since a further increase in initiator concentration would lead to primary radical termination. The decrease in grafting percentage at higher APS concentrations is attributable to the decrease in the molecular weight of graft PAN.

Figure 4 shows similar data for the grafting of
MMA. The molecular weight of graft PMMA decreases gradually with increasing APS concentration, on the other hand, the number of grafts increases and levels off at about 2. Comparison of Figure 4 with Figure 3 shows that the number of grafts and the grafting percentage differ greatly for the two monomer systems. The lower grafting percentage in the MMA-APS system is due to the lower number of grafts. Such differences in the number of grafts and the grafting percentage may be ascribed to the difference in monomer reactivity.

The increase in monomer concentration probably causes an increase in the molecular weight of graft polymer. ${ }^{24-26}$ Figures 5 and 6 show the effects of monomer concentration on the grafting of $\mathrm{AN}$ and MMA, respectively. The monomer concentration is expressed in grams of the monomer in $100 \mathrm{~g}$ of the DMSO solution. The initiator concentrations were chosen as $0.8 \mathrm{~g} / 100 \mathrm{~g}$ for the AN-APS system and $0.2 \mathrm{~g} / 100 \mathrm{~g}$ for the MMA-APS system, respectively. These values make possible the best grafting percentage and grafting efficiency for each monomer system.

As the monomer concentration is increased, the molecular weight of graft polymer increases monotonously, whereas the number of grafts decreases sharply at nearly the concentration where the grafting percentage is a maximum. The decrease in grafting percentage at higher monomer concentrations can be explained by the decrease in the number of grafts.

The solubility of cellulose in the DMSO-PF solvent system should become less by the addition of a monomer which is a nonsolvent for cellulose. Furthermore, the increase in monomer concentration facilitates the consumption of primary radicals to reduce the formation of cellulose macroradicals. These findings may possibly be an explanation for the decrease in the number of grafts at higher monomer concentrations.

A similar dependence of the number of grafts on monomer concentration has been reported for the grafting of styrene onto natural rubber in a homogeneous solvent system. ${ }^{27}$ On the other hand, it has been reported that the number of grafts does not depend on monomer concentration. ${ }^{8,28,29}$ Therefore, the dependence of the number of grafts on monomer concentration should be discussed for each grafting system. 


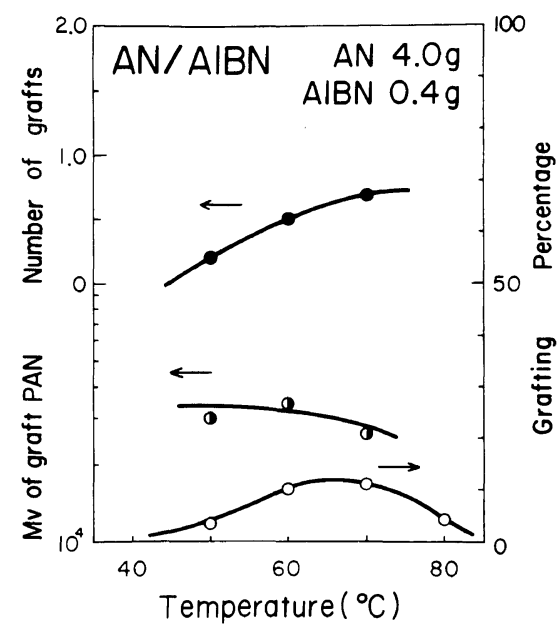

Figure 7. Effect of polymerization temperature on the grafting of AN: cellulose concn, $2 \%$; grafting time, $5 \mathrm{~h}$.

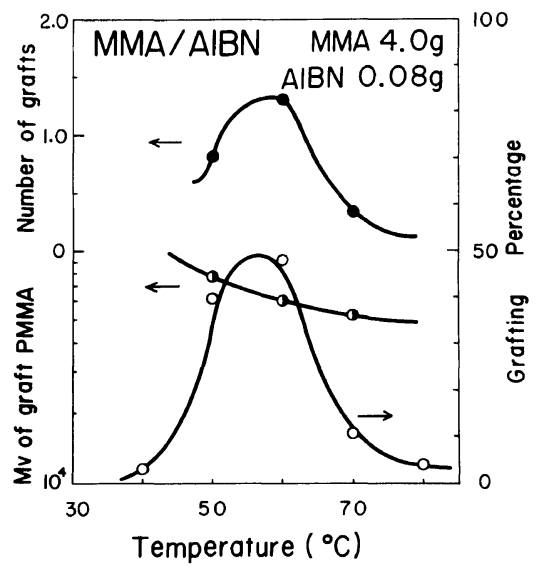

Figure 8. Effect of polymerization temperature on the grafting of MMA: cellulose concn, $2 \%$; grafting time, $5 \mathrm{~h}$.

\section{Initiation with Azobisisobutyronitrile}

It is well known that azobisisobutyronitrile (AIBN) is not suitable as a grafting initiator because of the resonance stabilization of its radical fragments. ${ }^{3}$ As indicated in our previous paper, ${ }^{10}$ however, grafting onto cellulose in the DMSO-PF solvent system showed that AIBN was effective for the grafting of MMA but not for that of AN.

The effect of temperature on the grafting of $\mathrm{AN}$ is shown in Figure 7. The grafting percentage is $10 \%$ at most, and no higher value was obtained, even though other grafting parameters were varied. The

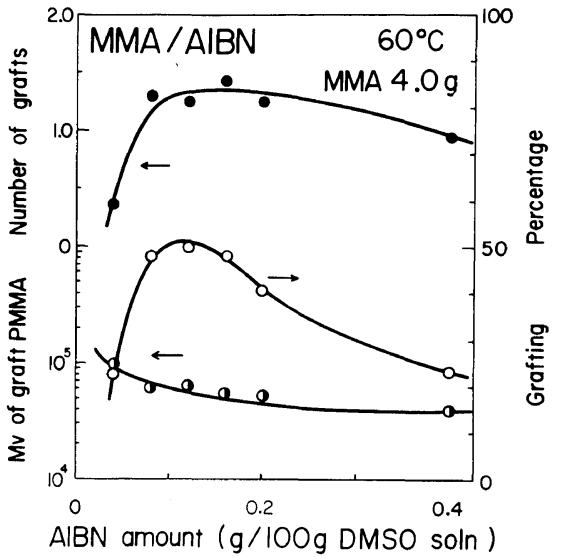

Figure 9. Effect of initiator concentration on the grafting of MMA: cellulose concn, $2 \%$, grafting time $5 \mathrm{~h}$.

molecular weight of graft PAN decreases gradually with raising temperature, whereas the number of grafts increases gradually but is less than unity. Comparison of this figure with Figure 1 shows that the grafting percentage and the number of grafts for the AN-AIBN system are much lower than those for the AN-APS system. This reveals that the chain transfer from the PAN homopolymer macroradicals to cellulose took place only slightly in this monomer-initiator system, because the chain transfer from AIBN primary radicals to cellulose may be negligible due to resonance stabilization.

Figure 8 shows the effect of temperature on the grafting of MMA. In contrast with the case of ANAIBN system, the grafting percentage and the number of grafts exhibit maxima around $60^{\circ} \mathrm{C}$, indicating that grafting took place effectively with MMA. Comparison of Figure 8 with Figure 2 reveals that the temperature dependence of the number of grafts differs remarkably. This trend cannot be explained by only an increase in the decomposition rate of the initiator.

Figure 9 shows the effect of initiator concentration on the grafting of MMA. The molecular weight of graft PMMA decreases gradually with increasing AIBN concentration. However, the number of grafts tends to decrease at higher AIBN concentrations. As mentioned above, cellulose macroradicals are formed primarily by the chain transfer from homopolymer radicals to cellulose in the AIBN system. In addition, the increase in radical concentration leads to the termination of 


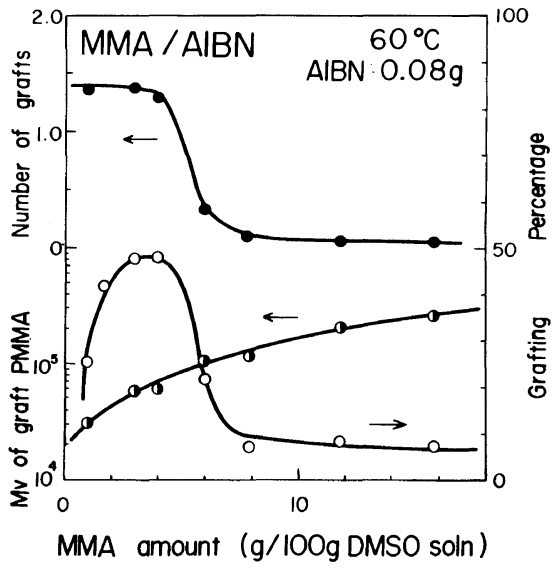

Figure 10. Effect of monomer concentration on the grafting of MMA: cellulose concn, $2 \%$; grafting time, $5 \mathrm{~h}$.

growing polymers with primary radicals. Thus the decrease in the number of grafts at higher AIBN concentrations may be attributed to the decrease in homopolymer macroradical concentration. This may also account for the number of grafts at higher temperatures shown in Figure 8.

Figure 10 shows the effect of monomer concentration on the grafting of MMA. The molecular weight of graft PMMA increases gradually with increasing MMA concentration. But the number of grafts decreases at higher MMA concentrations. These are the same tendencies as the results found in the MMA-APS system.

As discussed in relation to Figures 5 and 6, the decrease in the number of grafts at higher MMA concentrations may be due to the decrease in the solubility of cellulose in the DMSO-PF solvent system. Moreover, the increase in the molecular weight of the homopolymer makes difficult the chain transfer reaction from homopolymer macroradicals to cellulose. Thus, it seems reasonable to consider that the decrease in the number of grafts at higher MMA concentrations is due to the effect of the increase in homopolymer molecular weight rather than to that of the decrease in radical concentration.

\section{REFERENCES}

1. E. H. Immergut, "Encyclopedia of Polymer Science and Technology," Vol. 3, H. F. Mark, N. G. Gaylord, and N. M. Bikales, Ed., Interscience, New
York, 1965, p 242.

2. V. T. Stannett and H. B. Hopfenberg, "Cellulose and Cellulose Derivatives," Vol. V, Part V, N. M. Bikales and L. Segal, Ed., Wiley, New York, 1971, p 907.

3. F. Ide, "Graft Jugo to Sono Oyo," Kobunshi Kankokai, Kyoto, 1977.

4. S. M. Hudson and J. A. Cuculo, J. Macromol. Sci., Rev. Macromol. Chem., C18, 1 (1980).

5. I. Sakurada, Y. Ikada, and Y. Nishizaki, J. Polym. Sci., C, 37, 265 (1972).

6. T. Taga and H. Inagaki, Angew. Makromol. Chem., 33, 129 (1973).

7. Y. Ogiwara, H. Kubota, and K. Arai, J. Appl. Polym. Sci., 14, 1049 (1970).

8. P. Lepoutre and S. H. Hui, J. Appl. Polym. Sci., 19, 1257 (1975).

9. A. Moze and S. Lapanje, Makromol. Chem., 180, 1599 (1979).

10. N. Nishioka and K. Kosai, Polym. J., 13, 1125 (1981).

11. M. Tsuzuki, I. Hagiwara, N. Shiraishi, and T. Yokota, J. Appl. Polym. Sci., 25, 2909 (1980).

12. P. Cremonest, Cellulose Chem. Technol., 2, 459 (1968).

13. F. Ide, Kogyo Kagaku Zasshi, 64, 1489 (1961).

14. R. L. Cleland and W. H. Stockmayer, J. Polym. Sci., 17, 473 (1955).

15. H.-J. Cantow and G. V. Schulz, Z. Physik. Chem., 2, 117 (1954).

16. H. A. Swenson, J. Appl. Polym. Symp., 28, 945 (1976).

17. G. Jayme and P. Kleppe, Paper, 15, 492 (1961).

18. H. Yasuda, J. A. Wrag, and V. T. Stannett, J. Polym. Sci., C, 2, 387 (1963).

19. H. A. Ende and V. T. Stannett, J. Polym. Sci., A, 2, 4047 (1964).

20. T. I. Min and H. Inagaki, Polymer, 21, 309 (1980).

21. H. Inagaki, T. Kotaka, and T. I. Min, Pure Appl. Chem., 46, 61 (1976).

22. J. L. Lacatelli and G. Riess, Angew. Makromol. Chem., 28, 161 (1973).

23. J. L. Refgeggier, J. L. Lacatelli, and G. Riess, Eur. Polym. J., 10, 139 (1974).

24. Yo. Ogiwara, Yu. Ogawara, and H. Kubota, J. Polym. Sci., A-1, 5, 2791 (1967).

25. V. Hornof, B. V. Kokta, and J. L. Valade, J. Appl. Polym. Sci., 19, 545 (1975).

26. V. I. Kurliankina, V. A. Molotkov, S. I. Klenin, and S. Ya. Liubina, J. Polym. Sci., Polym. Chem. Ed., 18, 3369 (1980).

27. Y. Mori and Y. Minoura, Kogyo Kagaku Zasshi, 61, 263 (1958).

28. F. Ide, K. Nakatsuka, and H. Tamura, Kobunshi Kagaku, 23, 45 (1966).

29. K. Arai and M. Negishi, Sen-i Gakkai Shi, 23, 595 (1967). 